\title{
Global warming projections derived from an observation-based minimal model
}

\author{
K. Rypdal \\ Department of Mathematics and Statistics, UiT The Arctic University of Norway, Troms $\varnothing$, Norway \\ Correspondence to: K. Rypdal (kristoffer.rypdal@uit.no)
}

Received: 24 August 2015 - Published in Earth Syst. Dynam. Discuss.: 18 September 2015

Revised: 7 January 2016 - Accepted: 10 January 2016 - Published: 29 January 2016

\begin{abstract}
A simple conceptual model for the global mean surface temperature (GMST) response to $\mathrm{CO}_{2}$ emissions is presented and analysed. It consists of linear long-memory models for the GMST anomaly response $\Delta T$ to radiative forcing and the atmospheric $\mathrm{CO}_{2}$-concentration response $\Delta C$ to emission rate. The responses are connected by the standard logarithmic relation between $\mathrm{CO}_{2}$ concentration and its radiative forcing. The model depends on two sensitivity parameters, $\alpha_{T}$ and $\alpha_{\mathrm{C}}$, and two "inertia parameters," the memory exponents $\beta_{T}$ and $\beta_{\mathrm{C}}$. Based on observation data, and constrained by results from the Climate Model Intercomparison Project Phase 5 (CMIP5), the likely values and range of these parameters are estimated, and projections of future warming for the parameters in this range are computed for various idealised, but instructive, emission scenarios. It is concluded that delays in the initiation of an effective global emission reduction regime is the single most important factor that influences the magnitude of global warming over the next 2 centuries. The most important aspect of this study is the simplicity and transparency of the conceptual model, which makes it a useful tool for communicating the issue to non-climatologists, students, policy makers, and the general public.
\end{abstract}

\section{Introduction}

In spite of five comprehensive reports from the Intergovernmental Panel on Climate Change (IPCC), the perception of the threat of global warming to society remains highly diverse among the general public, decision makers, and the scientific community at large. This is in stark contrast to the general opinion among those who define themselves as climate scientists, where some studies suggest that as much as $97 \%$ recognise human activity as a main driver of global warming over the last century (Anderegg et al., 2010; Cook et al., 2013). What distinguishes the climate science community from other scientists is the strong reliance among climate scientists on complex earth system models (ESMs), that is, on atmospheric-ocean general circulation models (AOGCMs) coupled to models that include biogeochemistry and cryosphere dynamics. The general skepticism concerning this "model science" is not hard to understand. Models are complex beyond comprehension, different models are not independent but consist of many common modules, and parametrisations are empirical to an extent that makes it le- gitimate to question whether models are "massaged" to fit observations. The important point here is not whether this perception of climate modelling is correct or fair but that the skepticism exists and in many cases cannot be discarded as irrational.

The latest IPCC report from Work Group I on the climate system (IPCC AR5 WG1, 2013) contains a summary for policy makers that describes findings from observations and model studies, which many physical scientists find unconvincing and which is not a very easy read for the general public. The unconvincing part is the above-mentioned excessive reliance on complex computer models. Most scientists want to understand and to be convinced by simple fundamental principles matched against clear-cut observations. Decision makers and the informed layman want to see simple, clear alternatives for the future, not a myriad of incomprehensible scenarios labelled by acronyms that carry no meaning to non-experts.

A very readable and important paper on the "The closing door of climate targets" (Stocker, 2013) was published by 
the Co-Chair of Work Group I alongside the IPCC AR5 report, intended to demonstrate that as mitigation is delayed, climate targets formulated in international agreements become unattainable. The results were based on the physical assumption of a linear relationship between the cumulated carbon emissions and peak global warming in scenarios where the cumulative emission is bounded. This relationship, and the constant of proportionality, were justified empirically from numerical experiments performed on a large number of ESMs which incorporate the global carbon cycle (Allen et al., 2009; Matthews et al., 2009). Some readers, however, will find it unsatisfactory that they have to "believe" the models in order to accept the conclusion of the paper. As a former plasma physicist, who only relatively recently has taken up research in earth system dynamics and climate science, I am often confronted with questions from former colleagues of the following type: "For half a century we have tried to model the transport properties of a magnetically confined plasma for controlled thermonuclear fusion, and we still have not succeeded very well, even though the physical system is infinitely simpler than the climate. Why do you think these horrendously complex climate models perform any better?".

A major motivation for the present paper is to find ways to communicate with, and gain support from, the scientists who ask such questions. I do this by deriving results similar to those obtained in Stocker (2013) in a more transparent manner and without resorting to complex ESMs as the primary justification. The underlying assumptions are justified from observations, although supporting evidence from AOGCMs is also discussed. The conceptual models of the temperature and atmospheric carbon response are linear and simple enough to be understood by anyone with some background in elementary calculus and ordinary differential equations. The scenarios explored are idealised and the results presented in figures that should be comprehensible for readers without training in mathematics or physical sciences.

Section 2 describes and justifies the conceptual model. Section 3 presents projections for atmospheric $\mathrm{CO}_{2}$ concentration and global mean surface temperature (GMST) for some idealised $\mathrm{CO}_{2}$ emission scenarios: one which is very close to the "business as usual" Representative Concentration Pathway 8.5 (RCP8.5) scenario employed by the IPCC, and other scenarios which represent systematic emission reduction initiated at different times in the future. This section also discusses policy implications that may follow from these projections, and Sect. 4 summarises and concludes the paper. Six appendices elaborate on the physical interpretation and justification of the minimal model and on some mathematical aspects that may appear as paradoxes. This material is placed in appendices in order to avoid the interruption of the logical flow that leads to the main results. The Supplement contains data files and a well-documented Mathematica notebook with routines that allow readers to replicate and extend all results presented in the paper.

\section{The conceptual model}

A closed model for the evolution of the global mean surface temperature (GMST) could consist of (i) a model for the GMST anomaly response $\Delta T(t)$ to radiative forcing $F(t)$, (ii) a model for the evolution of $\Delta C(t)$, given the $\mathrm{CO}_{2}$ emission history $R(t)$, and (iii) a well-established constitutive relation between $F(t)$ and $\Delta C(t)$. This paper proposes extremely simple, linear models for the GMST response (i) and the $\mathrm{CO}_{2}$ concentration response (ii). Each depends on two parameters characterising the strength and the inertia (memory) of the response. In order to keep the model sufficiently simple for a reader to be able to trace the connection between driver and response and the effect of variation of model parameters, major simplifying assumptions are made. One is to neglect all radiative forcing other than $\mathrm{CO}_{2}$. Although the main reason for this is to maintain simplicity, it is justified by forcing estimates that conclude that the non- $\mathrm{CO}_{2}$ contributions tend to cancel out over the industrial period (IPCC AR5 WG1, 2013). Other important simplifications are linearity and stationarity.

\subsection{Linearity}

Global temperature has been found to respond quite linearly to forcing in general circulation models (Meehl et al., 2004), and as long as the climate system is far from a major tipping point, this linearity may also pertain to the response of atmospheric $\mathrm{CO}_{2}$ content to emissions. The effect of spacetime non-linearity is important primarily in variability on a scale smaller than the global scale. On the global scale the response function has an approximate power-law form that makes the system respond by a scale-invariant stochastic process to a white-noise driver. This scale invariance is characterised by a spectral exponent $\beta$, which gives rise to a powerlaw tail in the response function $G(s) \sim s^{\beta / 2-1}$, where $s$ is the time following an impulse in the forcing. The physical interpretation of such a response is that the climate system consists of a number of different interacting subsystems with different response times. There will be a maximum response time and hence there will be a cut-off of the power-law tail in the response function for $s$ larger than this maximal time constant. The justification, interpretation and implication of this picture is further discussed in the appendices.

\subsection{Stationarity}

The response functions are assumed to be translation invariant, i.e. $G\left(t, t^{\prime}\right)=G\left(t-t^{\prime}\right)$. This means that the GMST and the $\mathrm{CO}_{2}$ concentration respond the same way in a future climate as they do now. For the GMST this is a reasonable assumption as long as the global general circulation pattern remains the same, i.e. as long as the climate system does not encounter a major tipping point. Examples of such tipping points are the glacial-interglacial transitions or the 
Dansgaard-Oeschger events during the last ice age (Bender, 2013). During the present interglacial period, the Holocene, there was a similar tipping event about $8.2 \mathrm{kyr}$ ago. These events are believed to be associated with a sudden influx of freshwater into the North Atlantic from the North American Laurentide ice sheet and related changes in the overturning ocean circulation. A number of potential tipping elements have been identified associated with global warming in the present Holocene climate (Lenton et al., 2013). Among these are the complete disappearance of the Arctic sea ice, runaway melting of the Greenland and West Antarctic ice sheets, a radical change in the Atlantic thermohaline ocean circulation and the El Niño-Southern Oscillation, shifts in the Indian and the western African monsoons, and dieback of the Amazon and the boreal forests. Transitions associated with tipping elements of these types can change the global temperature response as well as the carbon-cycle response significantly. Even in the absence of tipping points, the stationarity assumption may be particularly wrong for the $\mathrm{CO}_{2}$ concentration, where, e.g., saturation effects in the ocean mixed layer and the land biosphere may reduce fluxes in a future climate. It also neglects the coupling between sea surface temperature and the $\mathrm{CO}_{2}$ flux, which will reduce the flux into the ocean in a warmer climate. However, experiments with carbon-cycle models subject to sudden $\mathrm{CO}_{2}$ injections into the atmosphere indicate that the response in the $\mathrm{CO}_{2}$ concentration can be described by a power-law response function. This response is not stationary in the sense that it will be the same for a new carbon release in a future climate, but it may give an adequate description of the response to the present global warming event. Further details are given in Sects. 2.3 and 2.4 and in the appendices.

\subsection{The temperature response}

The simplest physics-based model of the GMST response is the zero-dimensional, linearised energy balance model (EBM):

$$
\frac{d}{\mathrm{~d} t} \Delta T=-\frac{1}{\tau_{T}} \Delta T+\frac{S}{\tau_{T}} F .
$$

Here, $\tau_{T}$ is the time constant for the relaxation of the temperature anomaly and $S$ is the climate sensitivity. The model is often denoted the Budyko-Sellers model and was first proposed by Budyko (1969) and Sellers (1969). A simple derivation can be found in Rypdal (2012), where it is also pointed out that it is impossible to find a single time constant that describes adequately the response to forcing on all timescales. The reduction to a linear model from the nonlinear EBM with the full Stefan-Boltzmann radiation law is found in Appendix E. This model is not only used for reproducing the global temperature to known (deterministic) forcing but can also be formulated as a stochastic differential equation by introducing a noise component to the forcing $F(t)$, representing the stochastic energy flux from atmospheric weather systems to the ocean and land surface (Rypdal and Rypdal, 2014). The solution to this equation can be written as a convolution integral

$\Delta T(t)=\int_{0}^{t} G_{T}\left(t-t^{\prime}\right) F\left(t^{\prime}\right) \mathrm{d} t^{\prime}$,

with an exponential response function

$G_{T}(t)=\left(S / \tau_{T}\right) \exp \left(-t / \tau_{T}\right)$

The temperature response to a purely stochastic forcing, $\mathrm{i}$. e., $F(t)$ is represented as Gaussian white noise and is an Ornstein-Uhlenbeck stochastic process. In discrete time, this corresponds to a first-order autoregressive $(\mathrm{AR}(1))$ process. If Eq. (1) provides an adequate description, with $F(t)$ separated into a deterministic and a white-noise component, then the residual obtained after subtracting the deterministic response from the observed annual GMST record should be a realisation of an $\mathrm{AR}(1)$ process. The time constant and the climate sensitivity can be determined by a maximumlikelihood estimation, and in Rypdal and Rypdal (2014), they were estimated to be $\tau \approx 4.3$ years and $S \approx 0.32 \mathrm{~km}^{2} \mathrm{~W}^{-1}$. However, the sensitivity obtained is lower than that obtained from climate models, the fast response to volcanic eruptions is higher than in the observed record, and the residual does not conform well with an AR(1) process. Rypdal and Rypdal (2014) demonstrated that the residual is better described by a model for persistent, fractional Gaussian noise (fGn). Such a noise can be produced by Eq. (2) if the exponential response function is replaced by a power-law function

$G_{T}(t)=\alpha_{T} t^{\beta_{T} / 2-1}$,

where the memory exponent $\beta_{T}$ is in the interval $0<\beta_{T}<1$. It can be shown that this process has a power spectral density of the form $\sim f^{-\beta_{T}}$, where $f$ is the frequency (Beran, 1994). Hence, $\beta_{T}=0$ corresponds to white noise, while increasing $\beta_{T}$ signifies increasing degree of memory (or persistence) in the process. In this response model it replaces the time constant $\tau_{T}$ of the simple EBM. The parameter $\alpha_{T}$ replaces the climate sensitivity $S$. In Rypdal and Rypdal (2014) the magnitude of the parameters $\alpha_{T}$ and $\beta_{T}$ were estimated from the instrumental GMST record, revealing rather strong persistence $\left(\beta_{T} \approx 0.75\right)$. Similar values were also found in multiproxy data for the Northern Hemisphere, and in $\emptyset_{\text {st- }}$ vand et al. (2014), they were found in data from a number of millennium-long AOGCM simulations. The long power-law tail in the response function may be interpreted as an effect of thermal exchange between the surface (e.g. the ocean mixed layer) and other components of the climate system with a higher heat capacity (e.g. the deep ocean). A two-layer ocean energy balance model, for instance, yields a response function with two exponentials with different time constants. In Geoffroy et al. (2013), such a two-layer model was compared 

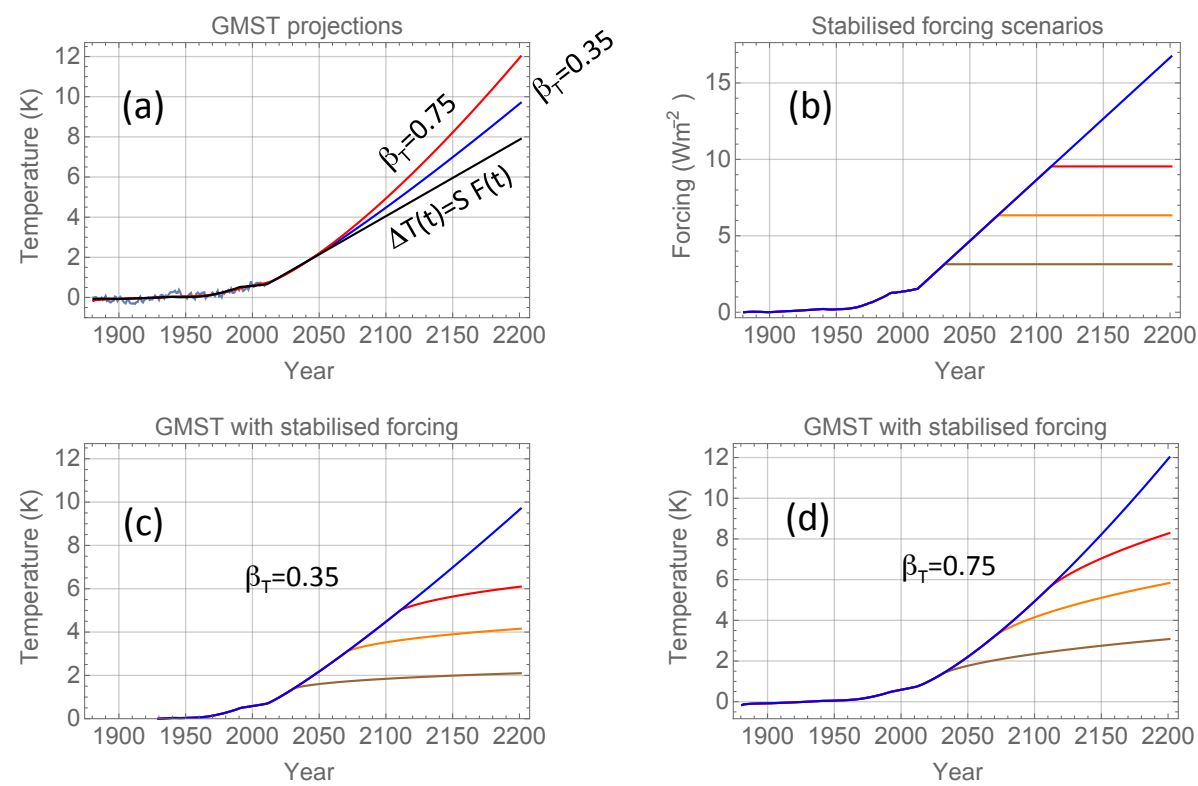

Figure 1. Panel (a): light blue curve is the instrumental GMST for 1880-2010 AD. Black curve is the instantaneous response to the linearly extrapolated forcing scenario shown in panel (b). Blue curve is the response according to the model Eq. (2) with $\beta_{T}=0.35$, and the red curve is the response with $\beta_{T}=0.75$. Panel (b): the straight sloping line is a linearly projected forcing to $2200 \mathrm{AD}$ with the same mean growth rate as the RCP8.5 scenario in the period 2010-2100 AD. The horizontal line is the stabilisation of this forcing in $2030 \mathrm{AD}$, the horizontal orange line in $2070 \mathrm{AD}$, and the red horizontal line in $2110 \mathrm{AD}$. Panel (c): GMST responses to the forcing scenarios in (b) with $\beta_{T}=0.35$. Colours correspond to those in (b). Panel (d): same as in (c) but with $\beta_{T}=0.75$.

to transient simulations of AOGCMs following an abrupt increase in $\mathrm{CO}_{2}$ forcing, and the two time constants estimated from these data were typically 1-2 years and 1-2 centuries. In Rypdal et al. (2015), it was shown that a power-law response provides an even better fit to the tail of the transient AOGCM solutions, but the memory exponent is lower ( $\beta_{T} \approx 0.35$ ) than that found from the residuals in observations and AOGCM simulations with historical forcing. One way of reconciling these conflicting results is to assume that the forcing noise is not white but rather a persistent noise, which makes a contribution to the $\beta_{T}$ observed in the residuals. Details are shown in Appendix D. On the other hand, it will be shown in Sect. 3 that the Coupled Model Intercomparison Project Phase 5 (CMIP5) in the RCP8.5 CO 2 concentration scenario yields results consistent with $\beta_{T}=0.75$. Since this implies some uncertainty with respect to the correct value of $\beta_{T}$ for the temperature response, I shall present projections for the values $\beta_{T}=0.35$ and $\beta_{T}=0.75$ in Sect. 3, assuming that $\beta_{T}$ is likely within this interval.

The significance of the inertia, or long-range memory (LRM), in the temperature response for GMST projections is illustrated in Fig. 1. Panel a shows the estimated GMST response to the forcing scenario consisting of the anthropogenic forcing in the period $1880-2010$ as presented in Hansen et al. (2011), linearly projected to $2200 \mathrm{AD}$ with the same mean growth rate as the RCP8.5 scenario in the period 2010-2100 AD (Meinshausen et al., 2011); it is shown as the blue curve in Fig. 1b. The blue and red curves in Fig. 1a are the responses according to the power-law response models with $\beta_{T}=0.35$, and $\beta_{T}=0.75$. The projection for an instant response ( $\tau_{T} \rightarrow 0$, leading to $\Delta T(t) \rightarrow S F(t)$ ) is also shown as the limit of zero inertia. Also shown as a light blue curve is the instrumental GMST record as given by Brohan et al. (2006). These projections have been obtained by computing the integral $\int_{0}^{t} \alpha_{T}\left(t-t^{\prime}\right)^{\left(\beta_{T} / 2-1\right)} F\left(t^{\prime}\right) \mathrm{d} t^{\prime}$ with the specified $\beta_{T}$ and then estimating $\alpha_{T}$ by regressing to the observed GMST record for the period 1880-2010 AD. The climate sensitivity $S$ for the instantaneous response has also been found by regressing $S F(t)$ to the instrumental data, and is found to be $S \approx 0.48 \mathrm{~km}^{2} \mathrm{~W}^{-1}$, which corresponds to $1.8 \mathrm{~K}$ for a doubling of $\mathrm{CO}_{2}$ concentration. The rising warming projected for increasing $\beta_{T}$ is a manifestation of the thermal inertia in parts of the climate system with high heat capacity that exchange heat with the surface, and it makes the surface temperature respond more slowly. The higher surface warming in the distant future due to this inertia is a manifestation of the warming in the pipeline (Hansen et al., 2011; Rypdal, 2012).

The forcing path represented by the straight sloping line shown in Fig. $1 \mathrm{~b}$ is an idealised business as usual (BAU) scenario. Beyond $2100 \mathrm{AD}$ there is every reason to believe that there will be a saturation of the rising trend, even in the absence of active mitigation policies. In the RCP8.5 this takes place gradually during the $22 \mathrm{nd}$ and the first half of the 
23rd century. This figure also shows some idealised scenarios where the BAU is modified by mitigation action. One possible type of action is the sudden reduction in emission that will stabilise the forcing at the level of the time of action. In the real world such an action from one year to another is not possible, but it may be considered an approximation of a certain annual reduction over a period of 1 decade. For instance, a $40 \%$ emission reduction can be achieved by an annual emission reduction of $5 \%$ over 1 decade. In Fig. 1b forcing scenarios for this type of mitigation action are illustrated assuming the onset of action in three different years: 2030, 2070, and 2110 AD. The year 2030 gives the world 15 years to prepare the action. Year 2070 leaves the problem to those who are born today, i.e. to the next generation. Year 2110 leaves it to unborn generations.

The GMST projections for these scenarios are shown in Fig. 1c, d for the lower and higher memory exponents $\beta_{T}$. Under the low-inertia assumption in the temperature response $\left(\beta_{T}=0.35\right)$, the unmitigated forcing scenario in Fig. 1a yields approximately $2{ }^{\circ} \mathrm{C}$ of warming every 40 years throughout the 21 st century and an even higher rate of warming in the 22nd century. After stabilisation of the atmospheric $\mathrm{CO}_{2}$ concentration, the temperature will continue to rise about $1^{\circ} \mathrm{C}$ by the year $2200 \mathrm{AD}$, independently of when this stabilisation takes place. This $1{ }^{\circ} \mathrm{C}$ of additional warming is the warming in the pipeline. Under the high-inertia assumption $\left(\beta_{T}=0.75\right)$, the warming rate is approximately $30 \%$ higher, and the warming in the pipeline is about a $100 \%$ higher. The high-inertia projection with mitigation action in $2110 \mathrm{AD}$ is very close to the multimodel mean RCP8.5 projection (Meinshausen et al., 2011), suggesting some consistency between this simple global temperature response model and the models employed by the IPCC in the CMIP5 project.

Figure $1 \mathrm{c}, \mathrm{d}$ suggest that the $2{ }^{\circ} \mathrm{C}$ target is unlikely to be attained by rapid stabilisation of atmospheric $\mathrm{CO}_{2}$ concentration if this action is started later than $2030 \mathrm{AD}$. If radical action is postponed until the GMST has passed the $2{ }^{\circ} \mathrm{C}$ limit, it is likely that the global temperature will exceed $3{ }^{\circ} \mathrm{C}$ by $2100 \mathrm{AD}$, and if action is postponed until the end of this century our descendants may experience a world that is $5-8{ }^{\circ} \mathrm{C}$ warmer than before industrialisation.

\subsection{The atmospheric $\mathrm{CO}_{2}$ response}

The dominant driver of climate change throughout the 20th century and beyond is anthropogenic radiative forcing, and in the 21st century, $\mathrm{CO}_{2}$ forcing is expected to be the main anthropogenic driver. However, while AOGCMs traditionally have been driven by prescribing the atmospheric $\mathrm{CO}_{2}$ concentration, the policy-relevant quantity is the $\mathrm{CO}_{2}$ emission rate. The main factor that determines future $\mathrm{CO}_{2}$ forcing in a given emission scenario is the rate at which $\mathrm{CO}_{2}$ is washed out of the atmosphere. This is where the carbon-cycle models incorporated into the ESMs become important. The model uncertainty is high, but the models suggest the existence of a hierarchy of timescales, just as we have found in the temperature response (Joos et al., 2011). This hierarchy is not immediately apparent from the instrumental data records, but there is some indirect evidence, as will be demonstrated below. However, let us first consider a primitive model with only one response timescale, analogous to the simple EBM given by Eq. (1) for the surface temperature. In this model we assume that the carbon flux out of the atmosphere is proportional to the anomaly $\Delta C$ of atmospheric carbon content relative to the preindustrial concentration $C_{0}$. This assumption follows from a Taylor expansion to first order of the carbon flux $I(\Delta C)=\left(1 / \tau_{\mathrm{c}}\right) \Delta C+\ldots$ around the preindustrial equilibrium $I\left(C_{0}\right)=0$. The primitive equation for this perturbation is then

$$
\frac{d}{\mathrm{~d} t} \Delta C=-\frac{1}{\tau_{\mathrm{C}}} \Delta C+R,
$$

where $\tau_{\mathrm{C}}$ is the time constant for the relaxation of $\mathrm{CO}_{2}$ concentration to the preindustrial equilibrium. A first-order estimate of $\tau_{\mathrm{C}}$ can be made from the estimates of the global carbon budget (Le Quéré et al., 2014). The annual carbon emission in the period 1960-2010 grew almost linearly from

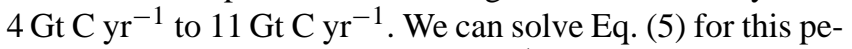
riod with $R=[4+(7 / 50)] t \mathrm{Gt} \mathrm{C} \mathrm{yr}^{-1}$ in terms of $\tau_{\mathrm{C}}$ and the initial atmospheric carbon inventory anomaly, $\Delta C_{1960}$. The conversion factor from concentration in part per million to gigatons of carbon in total carbon content is 2.12 (Le Quéré et al., 2014), which yields $\Delta C_{1960}=(315-280) \times 2.12 \approx 74 \mathrm{Gt}$ $\mathrm{C}$ if we assume a $\mathrm{CO}_{2}$ concentration of $315 \mathrm{ppm}$ in 1960 and a preindustrial concentration of $280 \mathrm{ppm}$. The preindustrial carbon content, corresponding to $280 \mathrm{ppm}$, was $C_{0} \approx 594 \mathrm{Gt}$ C. This solution reproduces very well the observed evolution of the atmospheric $\mathrm{CO}_{2}$ content in this period if one chooses $\tau_{\mathrm{C}}=33$ years, as shown in Fig. 2a, and suggests that $\Delta C(t)$ is described by the response function

$\Delta G_{\mathrm{C}}(t)=\left(r / \tau_{\mathrm{C}}\right) \exp \left[-t / \tau_{\mathrm{C}}\right]$.

A calibration factor $r$ has been introduced here because this response function is certainly too simplistic. For instance, Taylor expansion to first order does not take into account the saturation of carbon flux into the ocean, which will invoke a much longer response time governed by biogeochemical processes of the transport of carbon from the mixed layer into the deep ocean. If we fix $\tau_{\mathrm{c}}$ at value higher than 33 years, $r$ can be estimated by a simple, linear regression to the historic $\mathrm{CO}_{2}$ concentration record. For $\tau_{\mathrm{C}}=33$ years such regression yields of course $r \approx 1$ but for $\tau_{\mathrm{C}} \geq 300$ years, it yields $r \approx 0.5$. This means that the "effective emission rate" in Eq. (5) is reduced to $r R(t)$. The natural interpretation is that approximately half of the emitted $\mathrm{CO}_{2}$ is almost instantly removed from the atmosphere and the remainder has a lifetime of centuries, maybe millennia, i.e. that the response occurs on one fast and one slow timescale. Model studies, however, 

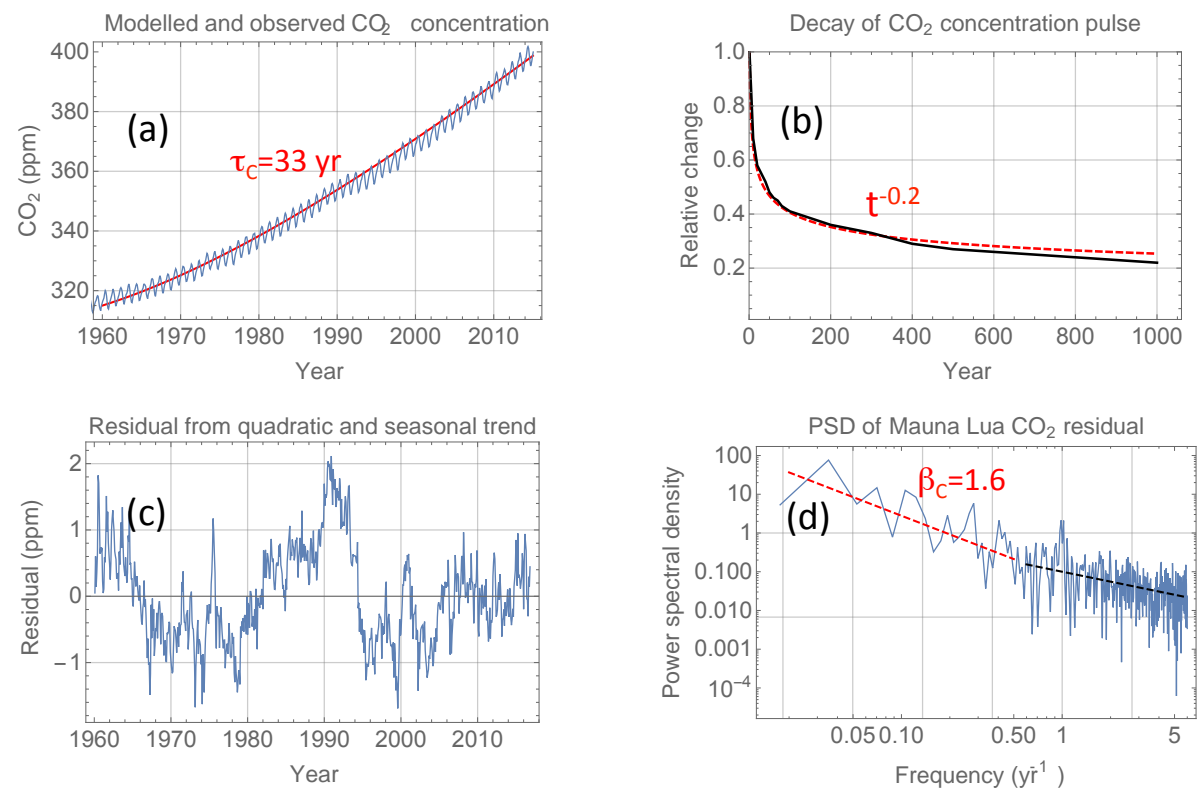

Figure 2. Panel (a): blue curve shows the atmospheric $\mathrm{CO}_{2}$ concentration as measured by the Mauna Loa observatory. The red curve is the concentration computed from Eq. (5) with $\tau_{\mathrm{C}}=33$ years, $\Delta C_{1960}=74 \mathrm{Gt} \mathrm{C}$ (corresponding to an anomaly of $315-280=35 \mathrm{ppm}$ ), and $C_{0}=594 \mathrm{Gt} \mathrm{C}$ (corresponding to $280 \mathrm{ppm}$ ). Panel (b): black curve is the multimodel mean $\mathrm{CO}_{2}$ response to a pulse of emitted $\mathrm{CO}_{2}$ as given in Joos et al. (2011). The red, dashed curve is a least-square fit of a function of the form $\alpha_{\mathrm{C}} t^{\beta_{\mathrm{C}} / 2-1}$ with the estimated $\beta_{\mathrm{C}} \approx 1.6$. Panel (c): the residual Mauna Loa signal after subtracting the quadratic polynomial and seasonal trends. Panel (d): the power spectral density of the residual in (c) estimated by the periodogram presented in a $\log -\log$ plot. The black, dashed line has negative slope $\beta_{\mathrm{C}}=0.85$, and the red, dashed line has $\beta_{\mathrm{C}}=1.6$.

may suggest a hierarchy of timescales for the $\mathrm{CO}_{2}$ concentration response. The large model comparison study of Joos et al. (2011) reveals a non-exponential tail in the response to a pulse of emitted $\mathrm{CO}_{2}$. Figure $2 \mathrm{~b}$ shows that the multimodel mean is very well approximated by a power law of the form

$G_{\mathrm{C}}(t)=\alpha_{\mathrm{C}} t^{\beta_{\mathrm{C}} / 2-1}$,

with $\beta_{\mathrm{C}} \approx 1.6$. This power-law response suggests the simple, linear response model

$\Delta C(t)=\int_{0}^{t} G_{\mathrm{C}}\left(t-t^{\prime}\right) R\left(t^{\prime}\right) \mathrm{d} t^{\prime}$,

where the emission rate $R(t)$ may contain a stochastic contribution, giving rise to a stochastic component to $\Delta C$. This stochastic component of $\Delta C$ is shown in Fig. 2c, as the residual obtained after subtracting a quadratic, polynomial fit to the Mauna Loa record (the anthropogenic trend) and the seasonal variation. The power spectral density of this residual is shown in Fig. $2 \mathrm{c}$ and indicates that the spectrum is consistent with a power law with a spectral index $\beta_{\mathrm{C}} \approx 1.6$ on timescales longer than a few years. The short duration of the record precludes accurate estimates of $\beta_{\mathrm{C}}$ from the spectrum, but it lends some support to the power-law response model with a memory exponent in the range $1<\beta_{\mathrm{C}}<2$.

\subsection{The constitutive relation}

A simple relation between $\mathrm{CO}_{2}$ concentration anomaly and its radiative forcing is (Myhre et al., 1998)

$F=5.35 \ln \left(1+\Delta C / C_{0}\right) \mathrm{Wm}^{-2}$.

Given an emission scenario $R(t)$, Eq. (8) can be used to compute $\Delta C(t)$, and from Eq. (9) one obtains $F(t)$. Finally, this forcing is applied in Eq. (2) to compute $\Delta T(t)$.

\section{Projections}

\subsection{Emission scenarios}

Figure 3 shows six different $\mathrm{CO}_{2}$ emission scenarios. The baseline (unmitigated) scenario is the blue curve, which is an exponential fitted with the actual emission rates in 1960 and in $2010 \mathrm{AD}$. Interpreted as $\mathrm{CO}_{2}$ equivalents of all well-mixed greenhouse gases, it is close to the RCP8.5 emission scenario up until 2070 but is higher after this time, since the RCP8.5 emission rates saturate between 2070 and 2100. At 2030, 2070 , and $2110 \mathrm{AD}$, two types of mitigation action are considered. One where emissions are reduced by $1 \%$ per year (50\% reduction over 70 years) and one with $5 \%$ per year (50\% reduction over 13.5 years). The former is considered politically and economically feasible (Stern, 2007); the latter is at the limit of what is possible without total disruption 


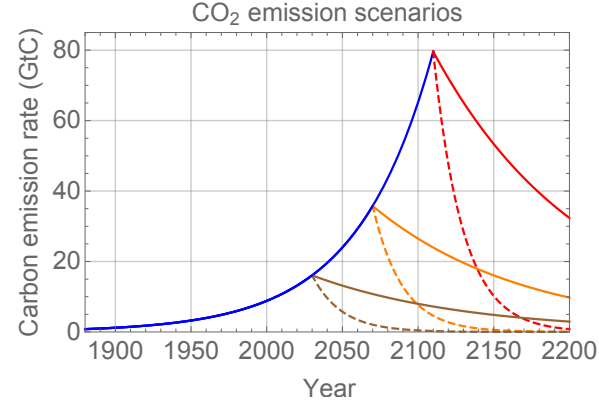

Figure 3. Blue curve is carbon emission rate $R(t)$ scenario obtained by fitting the exponential $S_{0} \exp (g t)$ to the emission rate $4 \mathrm{Gt} \mathrm{C} \mathrm{yr}^{-1}$ in 1960 and $11 \mathrm{Gt} \mathrm{C} \mathrm{yr}^{-1}$ in $2010 \mathrm{AD}$. The solid, brown, orange, and red curves are the subsequent $R(t)$ after initiation of a $1 \%$ reduction in the emission rate per year. The dashed curves are the corresponding rates with a $5 \%$ reduction per year.

of the world economy (Elzen et al., 2007). The scenarios are similar to those considered by Stocker (2013), although they are prescribed from $1880 \mathrm{AD}$, not from the present day. This is important for the response models employed here, since inertia (long-memory) effects from the historical period of global emissions and warming influence the future projections.

\subsection{Projections of $\mathrm{CO}_{2}$ concentration}

Atmospheric $\mathrm{CO}_{2}$ concentrations $\Delta C(t)$ for the emission scenarios described in Fig. 3 are shown in Fig. 4. They are computed from Eq. (8), using the emission scenarios of Fig. 3 and subsequently estimating $r$ by regressing to the historic $\Delta C(t)$ record. Figure 4a shows the corresponding concentration scenarios estimated from the exponential response kernel with $\tau_{\mathrm{c}}=33$ years. Few climate scientists believe that atmospheric, anthropogenic $\mathrm{CO}_{2}$ is eliminated as fast as this, but it is interesting to examine, since this is still claimed by some global warming skeptics (Solomon, 2008). In Fig. $4 \mathrm{~b}$ and $\mathrm{d}$ the same scenarios are shown, assuming $\tau_{\mathrm{c}}=300$ years and $\tau_{\mathrm{c}}=\infty$, respectively. Here $r \approx 0.5$, i.e. $50 \%$ of the emitted $\mathrm{CO}_{2}$, is immediately removed from the atmosphere and the rest decays exponentially with e-folding time $\tau_{\mathrm{C}}$. Figure $4 \mathrm{c}$ employs the power-law response kernel with $\beta_{\mathrm{C}}=1.6$. Figure $4 \mathrm{~b}$ and $\mathrm{c}$ are almost identical, indicating that the immediate removal of half of the emitted $\mathrm{CO}_{2}$, followed by an exponential decay with $\tau_{\mathrm{C}}=300$ years, has almost the same effect as a long-memory (power-law) response with $\beta_{\mathrm{C}}=1.6$.

The unmitigated concentration scenarios (blue curves) are almost the same in all models and are very similar to the RCP8.5 scenario up to $2100 \mathrm{AD}$. This is because the calibration factor $r$ adjusts the scenario to fit the historic record. However, the evolution after mitigation action has started varies considerably between the models. The overly optimistic model in Fig. $4 \mathrm{a}$, where $\tau_{\mathrm{C}}=33$ years, predicts that the concentration starts declining a few decades after emission reduction has started, whereas concentration continues to rise beyond $2200 \mathrm{AD}$ in the $1 \%$ reduction scenarios. The scenarios corresponding to the red solid curves in Fig. $4 \mathrm{~b}$ and c correspond closely to the full RCP8.5 scenario.

\subsection{Projections of the GMST}

The forcing $F(t)$ for the various concentration scenarios is computed from Eq. (9) and inserted into Eq. (2) to obtain the temperature evolution. Figure 5 shows results for the concentration scenarios obtained from the exponential $\mathrm{CO}_{2}$ concentration model with $\tau_{\mathrm{C}}=33$ years and the power-law model with $\beta_{\mathrm{C}}=1.6$, considering these to represent low- and highinertia ends of the $\mathrm{CO}_{2}$ response. For each of these cases, low- and high-inertia ends ( $\beta_{T}=0.35$ and $\left.\beta_{T}=0.75\right)$ of the GMST response are presented in the figure.

The projections for the high-inertia combination $\beta_{\mathrm{C}}=1.6$, $\beta_{T}=0.75$ shown in Fig. $5 \mathrm{~d}$ is the one that is most consistent with multimodel CMIP5 projections in the RCP8.5 scenario. As mentioned in Sect. 3.2, the red curve in Fig. 4c is close to the RCP8.5 $\mathrm{CO}_{2}$ concentration pathway, and the corresponding GMST response shown by the red curve in Fig. 5d is close to the multimodel mean GMST response given in Fig. 6 of Meinshausen et al. (2011). The high-end inertia $\left(\beta_{T}=0.75\right)$ for GMST response is also more consistent with the analysis of instrumental records and multiproxy reconstructions of GMST (Rypdal et al., 2015) and millennium-long simulations of intermediate and high complexity (Østvand et al., 2014). The high-end inertia for the $\mathrm{CO}_{2}$ response is also more consistent with complex carboncycle models, and the long-memory nature of the residual Mauna Loa record, as shown in Fig. 2d.

\subsection{Policy implications}

The range of the projections corresponding to given emission scenarios presented in Fig. 5a-d is much wider than the uncertainty of scientific knowledge reflected in the climate science literature. However, it may give an indication of the doubts which are quite common outside the climate science community. Among these are the belief that $\mathrm{CO}_{2}$ is removed from the atmosphere within decades (Solomon, 2008), and that the GMST relaxes to a new radiative equilibrium within a few years after a sudden perturbation of radiative forcing (Schwartz, 2007). Figure 5a presents projections which follow from these perceptions. Interestingly, the unmitigated projections up to $2110 \mathrm{AD}$ (blue curves) are almost identical in all panels in Fig. 5. Hence, the inertia in the responses has little influence on the unmitigated response to the BAU emission scenario, and uncertainty about the magnitude of the inertia parameters does not contribute much to uncertainty in the response to this scenario. Uncertainty in these parameters mainly plays a role for the projected effect of the emission reduction after action has been taken, as can be ob- 

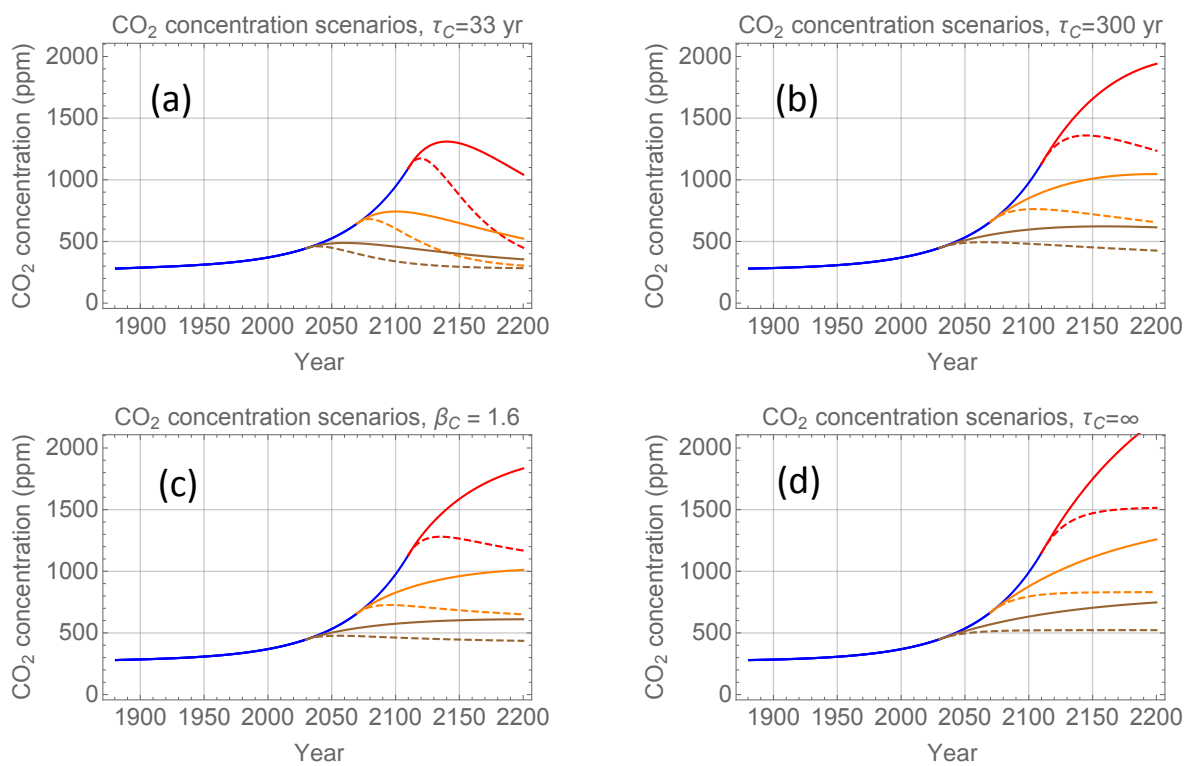

Figure 4. Projections of $\mathrm{CO}_{2}$ concentration under the emission scenarios in Fig. 3 using the modelling explained in Sect. 2. The colours correspond to those in Fig. 3. Panel (a): $\tau_{\mathrm{C}}=33$ years; panel (b): $\tau_{\mathrm{C}}=300$ years; panel (c): $\beta_{\mathrm{C}}=1.6$; panel $(\mathbf{d}): \tau_{\mathrm{C}}=\infty$.
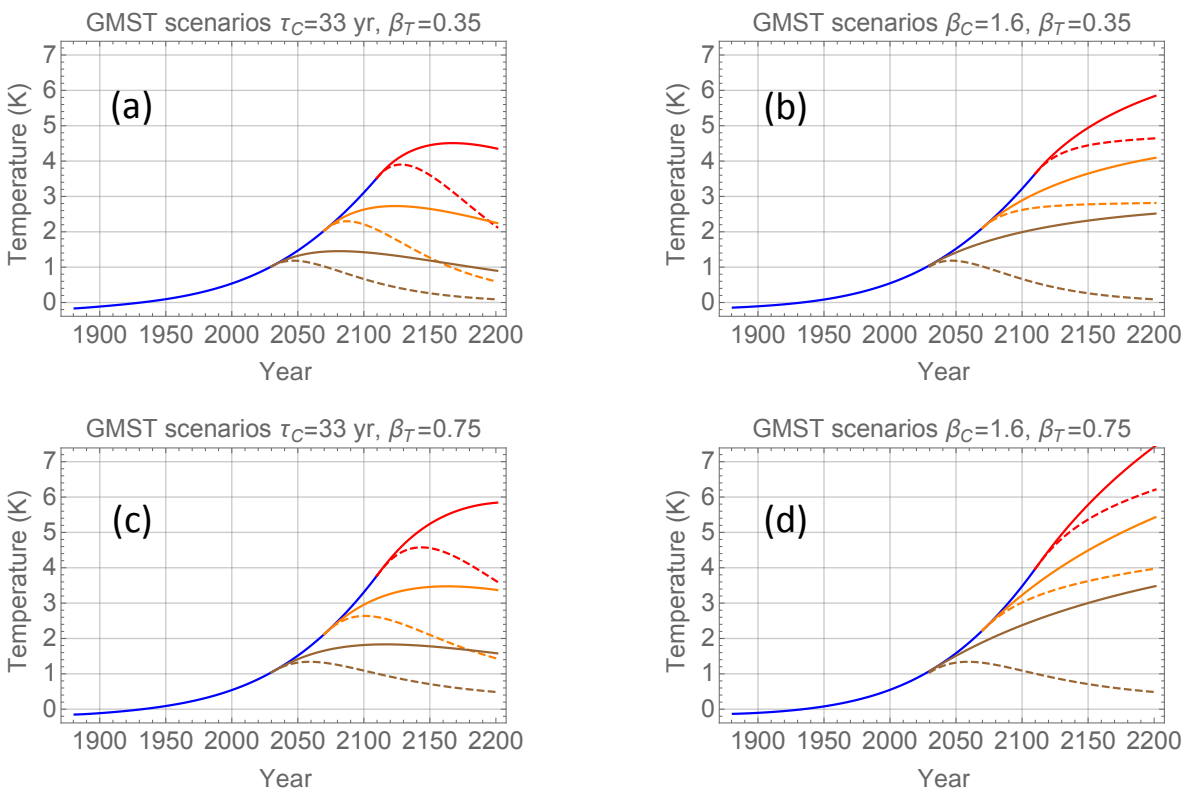

Figure 5. The evolution of the GMST for the $\mathrm{CO}_{2}$ concentration scenarios shown in Fig. $4 \mathrm{a}$ and $\mathrm{c}$. Panel (a): $\tau_{\mathrm{C}}=33$ years and $\beta_{T}=0.35$; panel (b): $\beta_{\mathrm{C}}=1.6$ and $\beta_{T}=0.35$; panel (c): $\tau_{\mathrm{C}}=33$ years and $\beta_{T}=0.75$; panel (d): $\beta_{\mathrm{C}}=1.6$ and $\beta_{T}=0.75$.

served by comparing Fig. 5a and d. The effect of emission reduction is considerably greater under the optimistic lowinertia assumptions, but in all circumstances, delayed mitigation action increases the GMST in $2200 \mathrm{AD}$ by $1-2{ }^{\circ} \mathrm{C}$ for every 40 years of delay.

One implication from this observation is that the global warming optimists have little reason for their optimism, since even the projections in Fig. 5a imply that the $2{ }^{\circ} \mathrm{C}$ climate tar- get will not be attained unless a radical and consistent emission reduction regime is initiated within a few decades from now. If this mitigation regime is delayed and initiated one generation later, even the optimistic projections indicate that the temperature will peak close to $3{ }^{\circ} \mathrm{C}$ during the next century, and postponing reduction for yet another generation will let the temperature rise beyond $4{ }^{\circ} \mathrm{C}$. If emission reductions are raised to the absolute threshold of $5 \%$ per year, the peak 
temperature will not change much, but the temperature will come down faster after action has been initiated.

Under the more pessimistic, and presumably more realistic, circumstances presented in Fig. $5 \mathrm{~b}$ and d, the $2{ }^{\circ} \mathrm{C}$ target is attainable only if extremely radical reductions $(5 \%$ per year) are initiated within the coming 2 decades. Since such a strong emission reduction regime probably is politically infeasible, this target most likely is unattainable, and the globe will warm $3-7^{\circ} \mathrm{C}$ before the end of next century. Where the GMST will end within this range will essentially depend on the time it takes before radical global emission reductions is implemented. Hence, the slow socio-economic response may turn out to be the most detrimental of all inertia effects which threaten to aggravate global warming.

\section{Conclusions}

It has been demonstrated that an extremely simple model for the global temperature response and the elimination of excess $\mathrm{CO}_{2}$ from the atmosphere is all that is needed to make reasonable projections of global temperature under idealised emission scenarios. The model contains only four parameters, characterising sensitivities and inertia in the temperature and $\mathrm{CO}_{2}$ responses, respectively. All parameters can be estimated from observation data, although some constraining from high-complexity ESMs is useful. The model can be used as a pedagogical tool for students and scientists with some knowledge of elementary calculus, and projections can easily be produced under emissions scenarios different from those presented here.
The simplicity of the model may be perceived as an insult to "real" climate modellers, but as long as one deals only with global quantities, simplicity does not necessarily mean lack of accuracy. Global temperature has been found to respond quite linearly to forcing in general circulation models (Meehl et al., 2004), and as long as the climate system is far from a major tipping point, this linearity may also pertain to the response of atmospheric $\mathrm{CO}_{2}$ content to emissions. Under linearity and stationarity assumptions these two quantities are fully described in terms of their respective response functions, whose form can be postulated from basic physical principles and parameters estimated from observation.

For the policy makers of the world it is crucial to know to what extent an economically and politically painful mitigation scenario can be expected to be effective in constraining global warming. The analysis presented here confirms the main conclusion drawn by Stocker (2013); the greatest threat to the stability of the global climate is the inability of humankind to respond in time. 
Appendix A: Response to step forcing for one-box model

The linearised one-box model has the form

$C_{1} \frac{d T_{1}}{\mathrm{~d} t}=-\frac{T_{1}}{S_{\mathrm{eq}}}+F$.

Here $T_{1}$ is the perturbation of the mixed-layer temperature from an imagined equilibrium and $F$ is the forcing relative to that equilibrium. $C_{1}$ is the heat capacity per square metre of the mixed layer, and the term $T_{1} / S_{\mathrm{eq}}$ is the linearised expression for the intensity of the outgoing long-wave radiation (OLR). It is determined by the (linearised) StefanBoltzmann (SB) law and the effective emissivity of the atmosphere, which also contains the effects of fast feedbacks. The nonlinear version and the linearisation procedure is described in Appendix E. If a new equilibrium is attained with the forcing $F$, we have

$S_{\text {eq }}=\frac{T_{1}}{F}$,

which makes it natural to identify $S_{\mathrm{eq}}$ as the equilibrium climate sensitivity. It is determined from the SB constant and the effective atmospheric emissivity, i.e. it is determined entirely by the atmosphere. The response function (Green's function: the response to $F=\delta(t)$ ) for the one-box model is $G(t)=\frac{1}{C_{1}} e^{-t / \tau_{1}} H(t)$, where $\tau_{1}=C_{1} S_{\text {eq }}$ and $H(t)$ is the Heaviside unit step function. The response to a step-function forcing $F(t)=H(t)$ is

$$
T_{1}(t)=\int_{-\infty}^{t} G\left(t-t^{\prime}\right) \mathrm{d} t^{\prime}=S_{\text {eq }}\left(1-e^{-t / \tau_{1}}\right)
$$

\section{Appendix B: Response to step forcing for two-box model}

The recent work by Geoffroy et al. (2013) shows that a twoexponential response can be fitted very well to a number of 150-year AOGCM runs with step-function forcing. This raises the question of whether the power-law LRM response representation is really only an inaccurate expression of a response with two exponential timescales or vice versa. There is also an issue of whether the AOGCMs really capture the true scaling properties of the observed response. The two-box model couples the mixed layer to the deep ocean temperature $T_{2}$ through a simple heat conduction term

$C_{1} \frac{d T_{1}}{\mathrm{~d} t}=-\frac{1}{S_{\mathrm{eq}}} T_{1}-\kappa\left(T_{1}-T_{2}\right)+F$

$C_{2} \frac{d T_{2}}{\mathrm{~d} t}=\kappa\left(T_{1}-T_{2}\right)$,

where $C_{2}$ is the heat capacity of the deep ocean and $\kappa$ is heat conductivity. In the limit $C_{2} \gg C_{1}$, Green's function for $T_{1}(t)$ correct to lowest order in the small parameter $C_{1} / C_{2}$ is very simple and transparent:

$G(t)=\left(\frac{S_{\mathrm{tr} 0}}{\tau_{\mathrm{tr}}} e^{-t / \tau_{\mathrm{tr}}}+\frac{S_{\mathrm{eq}}-S_{\mathrm{tr}}}{\tau_{\mathrm{eq}}} e^{-t / \tau_{\mathrm{eq}}}\right) H(t)$.

The response to a step-function forcing $F=H(t)$ then becomes

$T_{1}(t)=S_{\mathrm{tr}}\left(1-e^{-t / \tau_{\mathrm{tr}}}\right)+\left(S_{\mathrm{eq}}-S_{\mathrm{tr}}\right)\left(1-e^{-t / \tau_{\mathrm{eq}}}\right)$,

where we have introduced some new parameters,

$S_{\mathrm{tr}}=\frac{S_{\mathrm{eq}}}{1+\kappa S_{\mathrm{eq}}}, \tau_{\mathrm{tr}}=C_{1} S_{\mathrm{tr}}, \tau_{\mathrm{eq}}=\frac{C_{2} S_{\mathrm{eq}}}{1-S_{\mathrm{tr}} / S_{\mathrm{eq}}}$.

These parameters replace the heat capacities $C_{1,2}$ and the heat coupling constant $\kappa$, whose physical meaning is easy to grasp but hard to measure directly. The meaning of the new parameters is apparent if we consider the response to a step-function forcing. Since $C_{1} / C_{2} \ll 1$, we have $\tau_{\text {tr }} \ll \tau_{\text {eq }}$, and for $t \ll \tau_{\text {eq }}$ the response is completely dominated by the first term in Eq. (B3) and hence relaxes exponentially with the transient time constant $\tau_{\text {tr }}$ to the new quasi-equilibrium $S_{\text {tr }}$, which is referred to as the transient climate sensitivity. However, when $t$ approaches $\tau_{\text {eq }}$, the second term comes into play, and there is a new delayed response with time constant $\tau_{\text {eq }}$ giving relaxation to the full radiative equilibrium $S_{\text {eq }}$.

From comparing the terms $-T_{1} / S_{\text {eq }}$ and $-\kappa\left(T_{1}-T_{2}\right)$ in Eq. (B1), we observe that $\kappa S_{\mathrm{eq}}$ measures the ratio between the heat flux into the deep ocean and the OLR at the early stage of the response, i.e. when $T_{2}$ is still close to 0 . From Eq. (B4) it follows that the part of the sensitivity caused by the slow response from interaction with the deep ocean is

$S_{\mathrm{eq}}-S_{\mathrm{tr}}=\left(\kappa S_{\mathrm{eq}}\right) S_{\mathrm{tr}}$.

Hence, it appears that $\kappa S_{\mathrm{eq}}$ is an important parameter. If $\kappa S_{\text {eq }} \ll 1$, the inclusion of the deep ocean has little effect on the relaxation to equilibrium. If $\kappa S_{\mathrm{eq}} \simeq 1$ or larger, the slow response leads to a significant rise in the temperature after the transient equilibrium has been attained. The fast and the slow time constants are always well separated if $C_{1} \ll C_{2}$ since

$\frac{\tau_{\mathrm{tr}}}{\tau_{\mathrm{eq}}}=\frac{C_{1}}{C_{2}} \frac{\kappa S_{\mathrm{eq}}}{\left(1+k S_{\mathrm{eq}}\right)^{2}} \leq \frac{C_{1}}{4 C_{2}}$. 


\section{Appendix C: Response to step forcing in the LRM model and GCMs}

The LRM-scaling response function $G_{T}(t)=\alpha_{T} t^{\beta_{T} / 2-1}$ yields a response $T \sim t^{\beta_{T} / 2}$ to a step in the forcing at time $t=0$, while a linearly growing forcing yields a response $T \sim t^{\beta_{T} / 2+1}$. Since the forcing is logarithmic in the $\mathrm{CO}_{2}$ concentration, the latter corresponds to exponentially growing concentration. Climate model runs with linearly growing forcing are of course more realistic than step-function runs, but both have been conducted as part of the CMIP5 project. Examples are 150-year-long simulations of the GISS-E2-H model with a sudden quadrupling of the $\mathrm{CO}_{2}$ concentration (Fig. C1a) and a $1 \%$ per year increase in the $\mathrm{CO}_{2}$ concentration (Fig. C1b). A fit of the LRM-scaling response $T \sim t^{\beta_{T} / 2}$ to the GISS-model result in Fig. C1a yields $\beta_{T} \approx 0.32$, and the solution is shown as the red curve in the figure. The solution of the form $T \sim t^{\beta_{T} / 2+1}$ is shown as the red curve in Fig. C1b. The fit to the tail of the step-function response looks good in the 150-year duration of the simulation, but the divergence of the solution as $t \rightarrow \infty$ indicates that the powerlaw tail with $\beta_{T}>0$ is unrealistic for sufficiently large times. There exist few AOGCM simulations that investigate the response to such idealised forcing on a millennium timescale. In Hansen et al. (2011) some figures with results of such runs are given. Figure $\mathrm{Clc}$ is an adaptation of Fig. 3 in Hansen et al. (2011), which shows a 2000-year-long run of the GISS ModelE-R, and Fig. C1d shows a plot of the function $c t^{\beta_{T} / 2+1}$ with $\beta=0.32$. It demonstrates that at least this particular AOGCM exhibits the power-law tail in the temperature response on timescales of up to 2 millennia.
Note that the $\beta_{T} \approx 0.32$ obtained for the LRM model on long timescales is smaller than the $\beta_{T} \approx 0.75$ estimated from the spectra of the residual of the instrumental data after the response to the deterministic forcing has been subtracted (Rypdal and Rypdal, 2014). If we produce such residuals by subtracting the red curves from the GISS-model curves in Fig. $\mathrm{C} 1 \mathrm{a}, \mathrm{b}$, the result looks like fractional Gaussian noise (fGn) with a spectral exponent $\beta \approx 0.65$. As mentioned in Sect. 2.1 an fGn $x_{\beta}(t)$ characterised by the spectral exponent $\beta$ is produced by the convolution integral Eq. (2) in the main paper if the response kernel is $G(t) \sim t^{\beta / 2-1}$ and the forcing function $F(t)$ is white Gaussian noise $x_{0}(t)$ (white noise is an fGn with $\beta=0)$. In other words, we have

$x_{\beta}(t)=\int_{-\infty}^{\infty} t^{\prime \beta / 2-1} H\left(t-t^{\prime}\right) x_{0}\left(t^{\prime}\right) \mathrm{d} t^{\prime}$,

where $H(t)$ is the unit step function. By using the convolution theorem for the Fourier transform, it is easily shown (Rypdal et al., 2015) that if $F(t)$ is an fGn with spectral exponent $\beta_{F}$ and the response function has exponent $\beta_{T}$, then the convolution will produce an fGn with $\beta=\beta_{T}+\beta_{F}$ :

$x_{\beta}(t)=\int_{-\infty}^{\infty} t^{\beta^{\prime} / 2-1} H\left(t-t^{\prime}\right) x_{\beta_{F}}\left(t^{\prime}\right) \mathrm{d} t^{\prime}$.

In Rypdal et al. (2015) it was suggested that the discrepancy between the spectral exponent $\beta$ of residuals in observed and simulated GMST records could be explained by assuming some long-range memory $\left(\beta_{F}>0\right)$ in the stochastic forcing. It was pointed out there that this LRM could even be present in the $\mathrm{CO}_{2}$ forcing, since some recent studies indicate strong spatiotemporal heterogeneity in the atmospheric $\mathrm{CO}_{2}$ concentration, which might give rise to a fluctuating global component of the global $\mathrm{CO}_{2}$ forcing with long-memory properties. 

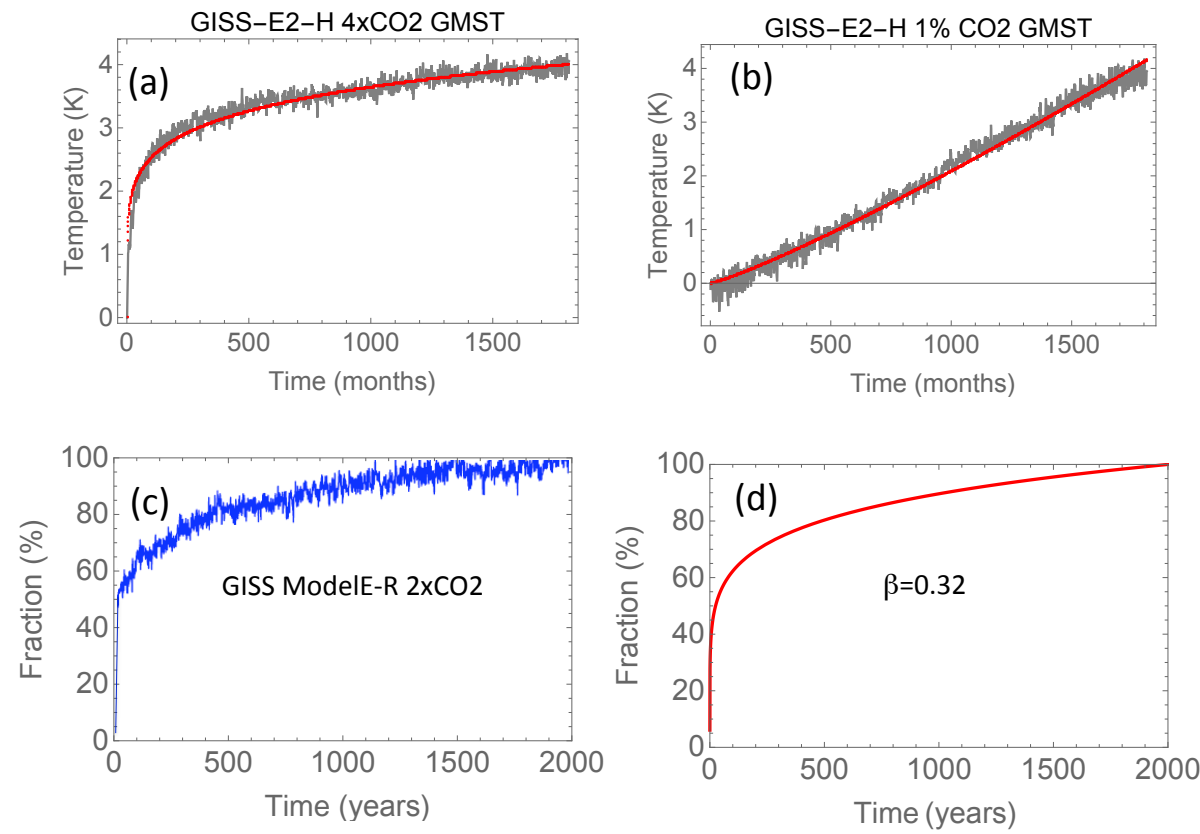

Figure C1. Panel (a): LRM response model fit $c_{1} t^{\beta_{T} / 2}$ (red) to the GISS-E2-H model response to an abrupt quadrupling of atmospheric $\mathrm{CO}_{2}$ (grey). The fit yields $\beta_{T}=0.32$. Panel (b): the LRM-response model solution $c_{2} t^{\beta_{T} / 2+1}$ with $\beta_{T}=0.32$ (red) and the GISS-E2-H model response to a $1 \%$ per year increase in atmospheric $\mathrm{CO}_{2}$ concentration. Panel (c): the 2000-year response to a doubling of $\mathrm{CO}_{2}$ in GISS ModelE-R as taken from Fig. 3 in Hansen et al. (2011). Panel (d): response to the same forcing in the LRM model with $\beta_{T}=0.32$. 


\section{Appendix D: Two-box vs. LRM fitting to GCM results}

Geoffroy et al. (2013) have considered 16 runs of different CMIP5 models with step-function forcing, and fitted the response in the two-box model to the CMIP5-model responses. There are four fitting parameters, and the fits are generally good. There is, however, a wide scatter in the fitting parameters between the different models, which may be an indication of overfitting. In Fig. D1 the surface temperature solution to the two-box model,

$T_{1}(t)=\left[S_{\mathrm{tr}}\left(1-\exp \left(-t / \tau_{\mathrm{tr}}\right)\right)\right.$

$\left.+\left(S_{\text {eq }}-S_{\text {tr }}\right)\left(1-\exp \left(-t / \tau_{\text {eq }}\right)\right)\right] F_{4 \times \mathrm{CO}_{2}}$,

and to the LRM model,

$T_{1}(t)=c t^{\beta_{T} / 2} F_{4 \times \mathrm{CO}_{2}}$,

have been fitted to simulation results for the GMST of climate models with step-forcing, $F(t)=F_{4 \times \mathrm{CO}_{2}} H(t)$. Here $F_{4 \times \mathrm{CO}_{2}} \approx 8.61 \mathrm{Wm}^{-2}$ is the forcing associated with a quadrupling of the atmospheric $\mathrm{CO}_{2}$ concentration. The fitting parameters obtained are given in Table 1.
The LRM model in general gives a poorer fit on the short timescales. This is not surprising, since the LRM response $c t^{\beta_{T} / 2}$ has an infinite derivative at $t=0$. However, a much better approximation is obtained if we fit the LRM model only in the interval $(0,100)$ months, but then $\beta_{T}$ is raised to approximately 0.75 . If we implement a four-parameter model with one power law $\left(\beta_{T} \approx 0.75\right)$ up to 100 months and another $\left(\beta_{T} \approx 0.35\right)$ for $t>100$ months, we obtain fits comparable to the two-exponential model. There is a wide scatter in the model parameters for the two-box model. Note particularly the huge values for $\tau_{\mathrm{eq}}$ and $S_{\mathrm{eq}}$ for the CCSM4 model. The long timescale tail is not captured by a reasonable exponential but is well approximated by a reasonable power law. On the other hand, the scatter in the LRM-model parameters is small. All this indicates that the two-box model may suffer from overfitting in some cases.

When projections are limited to $2200 \mathrm{CE}$, there is no practical difference between using a power-law response kernel (the LRM model) and the two-exponential kernel (the twobox model). This is illustrated in Fig. D2, where we compute the response for the exponential $\mathrm{CO}_{2}$ concentration model with $\tau_{\mathrm{C}}=33$ years and the two-box model parameters corresponding to the GISS-E2-H model and the CNRM_CM5 models. The parameters for the two models differ significantly, but the projections are almost identical. Moreover, they are very similar to the projections in Fig. 5a, where the temperature response is produced by the LRM model with $\tau_{\mathrm{C}}=33$ years and $\beta_{T}=0.35$. This demonstrates that the mathematical divergence of the solution Eq. (D2) for a step-function forcing has little impact on the projection up to $2200 \mathrm{CE}$ for the forcing scenarios considered here. The advantage of the power-law kernel is that it provides a more parsimonious description (fewer fitting parameters), which provides a more precise parameter estimation. 
Table D1. Parameters estimated by fitting Eqs. (D1) and (D2) to the climate model responses to an abrupt quadrupling of atmospheric $\mathrm{CO}_{2}$ shown in Fig. D1. The table shows the parameters obtained by the Mathematica routine FindFit.

\begin{tabular}{llllllr}
\hline Model & $\tau_{1}$ (Months) & $\tau_{2}$ (Months) & $S_{\text {tr }}\left(\mathrm{km}^{2} \mathrm{~W}^{-1}\right)$ & $S_{\mathrm{eq}}\left(\mathrm{km}^{2} \mathrm{~W}^{-1}\right)$ & $c$ & $\beta_{T}$ \\
\hline GISS-E2-H & 26 & 663 & 0.29 & 0.46 & 0.14 & 0.32 \\
BNU-ESM & 46 & 729 & 0.46 & 0.69 & 0.21 & 0.33 \\
CCSM4 & 49 & $4.1 \times 10^{10}$ & 0.33 & $3.9 \times 10^{6}$ & 0.10 & 0.40 \\
CNRM_CM5 & 38 & 390 & 0.37 & 0.58 & 0.20 & 0.31 \\
MPI-ESM-LR & 34 & 1061 & 0.46 & 0.75 & 0.20 & 0.33 \\
\hline
\end{tabular}
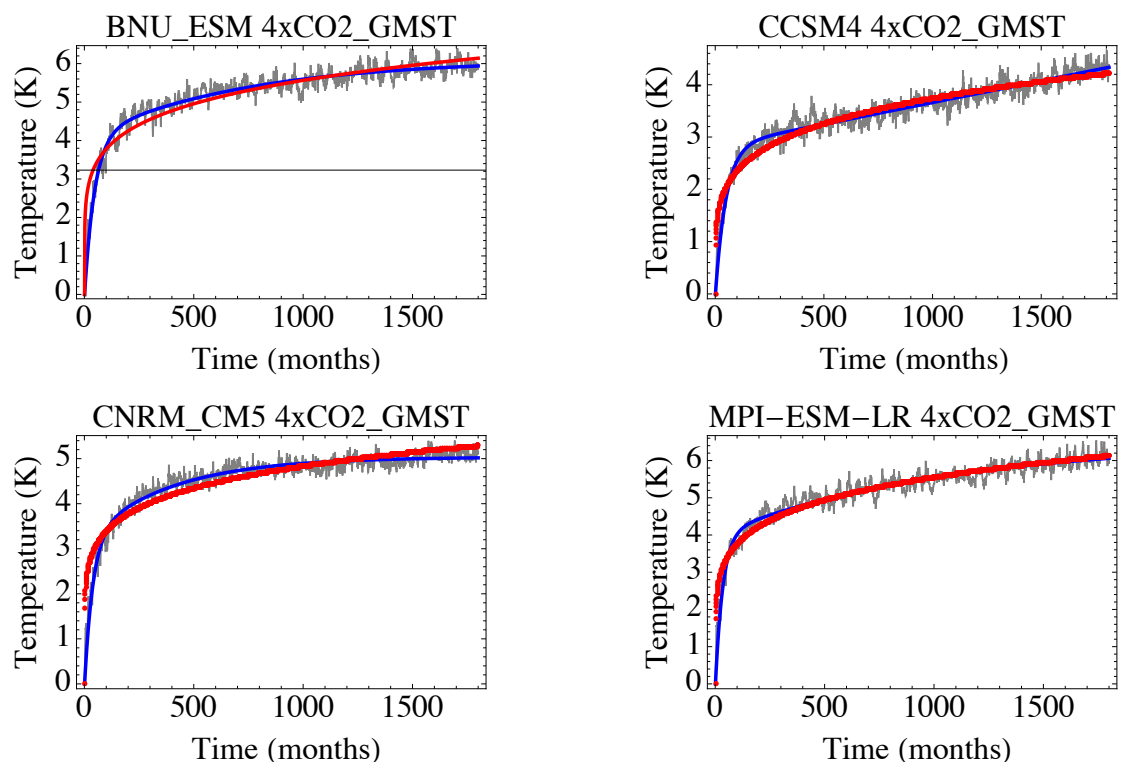

Figure D1. Blue curves: fit of the two-exponential response to the climate model responses to an abrupt quadrupling of atmospheric $\mathrm{CO}_{2}$ concentration. Red curves: fit of the LRM-scaling response. The expressions fitted are found in the caption of Table 1 and the coefficients estimated are shown in this table.
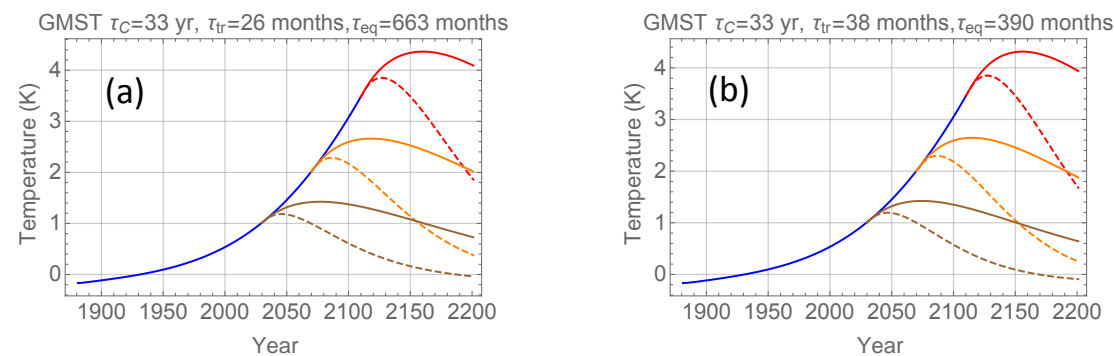

Figure D2. The evolution of the GMST according to the two-box model for the $\mathrm{CO}_{2}$ concentration scenarios shown in Fig. $4 \mathrm{a}$ and c. Panel (a): $\tau_{\mathrm{C}}=33$ years and the two-box parameters for the GISS-E2-H given in Table 1. Panel (b): $\tau_{\mathrm{C}}=33$ years and the two-box parameters for the CNRM_CM5 model given in Table 1 . 


\section{Appendix E: Divergences, causality and initial conditions}

If $G(t)$ is a power law, the integral over prehistory $t \in$ $(-\infty, 0)$ may lead to paradoxes, such as divergences of the integral. The solution to the paradox is to interpret the power law as an approximation, for instance to a superposition of exponential response kernels. For a white-noise forcing this corresponds to an aggregation of Ornstein-Uhlenbeck (OU) processes, which are known to have the potential to produce a process that is a very good approximation to a fractional Gaussian noise (fGn) up to the timescale corresponding to the OU process with the greatest correlation time (Granger, 1980).

The scaling properties on scales of decades and longer arise from the heat transport within the oceans. This transport exhibits a maximum response time, which will provide an upper (exponential) cut-off of the power-law response function, but the characteristic time of this cut-off may be centuries or millennia. Fraedrich and Blender (2003) state in their abstract: "Scaling up to decades is demonstrated in observations and coupled atmosphere-ocean models with complex and mixed-layer oceans. Only with the complex ocean model the simulated power laws extend up to centuries."

If we do not treat the power law as an approximation, we have to deal with the divergences of the integral

$$
\Delta T(t)=\int_{-\infty}^{t} G\left(t-t^{\prime}\right) F\left(t^{\prime}\right) \mathrm{d} t^{\prime},
$$

where $G(s)=s^{\beta_{T} / 2-1}$. If we consider the unit step-function forcing $F(t)=H(t)$ and $\beta_{T} \neq 0$, the integral is

$$
\begin{aligned}
& \Delta T(t)=\lim _{\epsilon \rightarrow 0^{+}} \int_{\epsilon}^{t}\left(t-t^{\prime}\right)^{\beta_{T} / 2-1} \mathrm{~d} t^{\prime}=\lim _{\epsilon \rightarrow 0^{+}} \int_{\epsilon}^{t} s^{\beta_{T} / 2-1} \mathrm{~s} \\
& =\lim _{\epsilon \rightarrow 0^{+}} \frac{2}{\beta_{T}}\left(t^{\beta_{T} / 2}-\epsilon^{\beta_{T} / 2}\right) .
\end{aligned}
$$

Clearly $\Delta T(t)$ diverges as $t \rightarrow \infty$ if $\beta_{T}>0$, but it also diverges if $\beta_{T}<0$ (as $\epsilon \rightarrow 0^{+}$). For $\beta_{T}=0$ there is a logarithmic divergence in both limits.

For physically meaningful results the $\beta_{T}>0$ case requires some sort of cut-off (e.g. an exponential tail) for sufficiently large $t$, and the $\beta_{T}<0$ case requires an elimination of the strong singularity of $G(s)$ at $s=0$. As shown in Appendix D, AOGCMs in the CMIP5 ensemble with step-function forcing indicate a power-law response for large $s$ at least up to 150 years (and the GISS-E2-R model up to 2000 years) with $\beta_{T} \approx 0.35$, so $\beta_{T}>0$ is the case of interest for the global temperature response. The AOGCMs are also well approximated by an exponential response in the limit $s \rightarrow 0$ (for $s$ up to a few years), so an exponential truncation in this highfrequency limit is also appropriate.
The truncation of the power-law kernels is a physical, and not a technical mathematical issue. It is an approximation to a hierarchy of exponential responses. With this interpretation the divergences evaporate. Below is a more detailed outline of this philosophy in an energy balance context. Let us take as a starting point the simple zero-dimensional EBM before linearisation of the Stefan-Boltzmann law:

$C \frac{\mathrm{d} T}{\mathrm{~d} t}=-\epsilon \sigma_{S} T^{4}+I(t)$,

where $T$ is surface temperature in Kelvin, $C$ is an effective heat capacity per area of the earth's surface, $\sigma_{S}$ is the Stefan-Boltzmann constant, $\epsilon$ is an effective emissivity of the atmosphere, and $I(t)$ is the incoming radiative flux density at the top of the atmosphere. Let $I_{0}=I(0)$ be the initial incoming flux, $F(t)=I(t)-I_{0}$ is the radiative forcing, $T_{\mathrm{eq}}=\left(I_{0} / \epsilon \sigma_{S}\right)^{1 / 4}$ is the equilibrium temperature at $t=0$, $\Delta T(t)=T(t)-T_{\mathrm{eq}}$ is the temperature anomaly measured relative to the initial equilibrium temperature, and $\Delta T_{0}=$ $\Delta T(0)$ is this anomaly at $t=0$. Note that $F$ here is the perturbation of the radiative flux with respect to the initial flux $I_{0}$ and not with respect to the flux $\epsilon \sigma_{S} T_{0}^{4}$ that would be in equilibrium with the initial temperature $T_{0}$. The linearised EBM for the temperature change relative to the temperature $T_{\text {eq }}$ (the one-box model) is

$\frac{d \Delta T}{\mathrm{~d} t}=-v \Delta T+\mathcal{F}(t), \quad \Delta T(0)=\Delta T_{0}$,

where $v=4 \epsilon \sigma_{S} T_{\text {eq }}^{3} / C, \quad \mathcal{F}(t)=F(t) / C$. By definition $\mathcal{F}(0)=\left[I(0)-I_{0}\right] / C=0$. This is Eq. (1) and Eq. (A1) with slightly different notation. The solution to the initial value problem (i.v.p.) Eq. (E4), with the initial condition $\Delta T(0)=\Delta T_{0}$, takes the form

$\Delta T_{\text {i.v.p. }}=\int_{0}^{t} G\left(t-t^{\prime}\right) \mathcal{F}\left(t^{\prime}\right) \mathrm{d} t^{\prime}+\Delta T_{0} e^{-v t}$,

where $G(s)=\exp (-v s)$. The generalisation to a linear, causal response model, where $G(s)$ is not necessarily exponential, involves extending the integration domain in Eq. (E5) to the interval $(-\infty, t)$ :

$\Delta T_{\text {r.m. }}(t)=\int_{-\infty}^{t} G\left(t-t^{\prime}\right) \mathcal{F}\left(t^{\prime}\right) \mathrm{d} t^{\prime}$.

From the initial condition $\Delta T(0)_{\text {r.m. }}=\Delta T_{0}$ Eq. (E6) yields

$$
\Delta T_{0}=\int_{-\infty}^{0} G\left(-t^{\prime}\right) \mathcal{F}\left(t^{\prime}\right) \mathrm{d} t^{\prime} .
$$

For exponential response $G(s)=\exp (-v s)$, it is easy to verify that $\Delta T_{\text {i.v.p. }}(t)=\Delta T_{\text {r.m. }}(t)$, and Eq. (E7) yields the following relation between the initial temperature anomaly and 
the forcing $\mathcal{F}(t)$ for $t \in(t, 0)$ :

$$
\Delta T_{0}=\int_{-\infty}^{0} e^{v t^{\prime}} \mathcal{F}\left(t^{\prime}\right) \mathrm{d} t^{\prime}
$$

For the exponential response there is no "divergence issue" in Eq. (E6). Neither is there such an issue for the twoexponential solution to the two-box model (Geoffroy et al., 2013). An " $N$-box model" exhibits a response function for the temperature in each box which is a superposition of exponentials; $G(s)=\sum_{i=1}^{N} a_{i} \exp \left(-v_{i} s\right)$. For the surface (mixed layer) box the temperature anomaly takes the form

$$
\Delta T_{\text {r.m. }}(t)=\sum_{i=1}^{N} a_{i} e^{-v_{i} t} \int_{-\infty}^{t} e^{v_{i} t^{\prime}} \mathcal{F}\left(t^{\prime}\right) \mathrm{d} t^{\prime}
$$

On the other hand, the $N$-box initial value problem has a solution of the form

$$
\Delta T_{\text {i.v.p. }}(t)=\sum_{i=1}^{N} a_{i} e^{-v_{i} t} \int_{0}^{t} e^{v_{i} t^{\prime}} \mathcal{F}\left(t^{\prime}\right) \mathrm{d} t^{\prime}+\sum_{i=1}^{N} b_{i} e^{-v_{i} t},
$$

where the coefficient $b_{i}$ is linearly related to the initial temperatures of each box: $b_{i}=\sum_{j=1}^{N} M_{i j} T_{0 j}$. The condition $\widetilde{T}_{\text {i.v.p. }}(t)=\widetilde{T}_{\text {r.m. }}(t)$ now yields the relations between the initial temperatures and the prehistory of the forcing:

$$
\sum_{j=1}^{N} M_{i j} \Delta T_{0 j}=a_{i} \int_{-\infty}^{0} e^{v_{i} t^{\prime}} \mathcal{F}\left(t^{\prime}\right) \mathrm{d} t^{\prime} \text { for } i=1, \ldots, N
$$

With a white-noise forcing $\mathcal{F}(t)$, Eq. (E4) is the Itô stochastic differential equation (in physics often called the Langevin equation). The solution is the Ornstein-Uhlenbeck (OU) stochastic process, which in discrete time corresponds to the first-order autoregressive (AR(1)) process. The power spectral density of this process is essentially a Lorentzian function, which means that the high-frequency $(f \gg v)$ part of the spectrum has the form $\sim f^{-2}$ and the low-frequency part $\sim f^{0}$. This means that if the climate response were well described by a one-box EBM we could use a power-law response model with $\beta_{T} \approx 2$ on timescales much shorter than the correlation time $\tau_{\mathrm{c}}=v^{-1}$. On these timescales the stochastic process exhibits the characteristics of a Brownian motion (Wiener process), which is a self-similar process with spectral index $\beta=2$. This process is non-stationary and hence suffers from the divergences that we are worried about. However, even though the Brownian motion diverges, the OU process does not because of the flattening of the spectrum for $f \ll v$.
Both observation data and AOGCMs indicate that the onebox EBM is inadequate, but the considerations above are equally valid for an $N$-box model, for which the white-noise forcing gives rise to an aggregation of OU processes with different $v_{i}$. Such an aggregation is known to be able to produce a process with an approximate power-law spectrum with $0<\beta<2$ on timescales $\tau<v_{\text {min }}^{-1}$ (Granger, 1980).

Lovejoy et al. (2013) specifically argue that volcanic forcing may have a scaling exponent $\beta_{F} \approx 0.4$, and hence the convergence criterion $\beta=\beta_{T}+\beta_{f}<1$ then requires $\beta_{T}<$ 0.6. One remark on this is that the above discussion shows that the $\beta<1$ criterion is not necessary on timescales shorter than $\tau<v_{\min }^{-1}$. However, observation indicates that $\beta<1$, so this does not invalidate the argument of Lovejoy et al. (2013). More important is that in recent papers the response to volcanic forcing has been subtracted from both instrumental and multiproxy reconstruction data (Rypdal and Rypdal, 2014) and from millennium-long AOGCM simulations ( $\varnothing$ stvand et al., 2014), and the residuals have been analysed for $\beta$ without finding a detectable influence of the volcanic forcing on $\beta$. The same is seen by comparing control runs of the AOGCMs with those driven by volcanic forcing (Østvand et al., 2014).

The importance of including the prehistory of the energyflux imbalance when deriving projections for future change can be illustrated by considering a prehistory consisting of volcanic forcing $\mathcal{F}_{V}(t)$ only. The particular feature of volcanic forcing is that it consists of a succession of negative spikes in the radiation flux. If we assume that the time $t=0$ is in a period with no volcanic forcing, we can for illustration think of the forcing as a succession of negative forcing events of short duration, randomly distributed in time with typically longer waiting times between events than durations. Let us further assume that the climate response is so slow that $G(t)$ varies by a small amount over the mean waiting time. Hence, there exist time intervals of duration $\Delta t$ which are short enough for $G(t)$ to be nearly constant over the interval but long enough to have a sufficient number of large volcanic eruptions to estimate a mean volcanic forcing $\overline{\mathcal{F}}_{V}$. This assumption is not very good in practice, but let us use it for illustration. Under this assumption we can approximate the integral

$$
\begin{aligned}
& \int_{t_{1}-\Delta t / 2}^{t_{1}+\Delta t / 2} G\left(t-t^{\prime}\right) \mathcal{F}_{V}\left(t^{\prime}\right) \mathrm{d} t^{\prime} \approx G\left(t-t_{1}\right) \int_{t_{1}-\Delta t / 2}^{t_{1}+\Delta t / 2} \mathcal{F}_{V}\left(t^{\prime}\right) \mathrm{d} t \\
& =G\left(t-t_{1}\right) \overline{\mathcal{F}}_{V} \Delta t,
\end{aligned}
$$

and hence from Eq. (E6) the temperature anomaly due to the volcanic forcing is 


$$
\begin{aligned}
& \Delta T_{V}(t)=\overline{\mathcal{F}}_{V} \int_{-\infty}^{t} G\left(t-t_{1}\right) \mathrm{d} t_{1} \\
& =\overline{\mathcal{F}}_{V} \int_{0}^{\infty} G(s) \mathrm{d} s \stackrel{\text { def }}{=}-\Delta T_{\text {volc }} .
\end{aligned}
$$

This result is meaningful only if the integral $\int_{0}^{\infty} G(s) \mathrm{d} s$ is finite, i.e. if power-law response kernels are properly truncated. The obvious, but still interesting, observation is that volcanic forcing keeps the temperature, when averaged over the timescale $\Delta t$, on a constant level $T_{\text {eq }}-\Delta T_{\text {volc }}$, i.e. the time-averaged temperature is $\Delta T_{\text {volc }}$ lower than the temperature at which the climate system is in equilibrium during times with no volcanic forcing.

Assume some additional (e.g. anthropogenic) forcing $\mathcal{F}_{A}(t)$, for which $\mathcal{F}_{A}=0$ for $t \leq 0$. Then the total temperature anomaly for $t>0$ would be

$$
\begin{aligned}
& \Delta T(t)=\Delta T_{V}(t)+\Delta T_{A}(t) \\
& =-\Delta T_{\text {volc }}+\int_{0}^{t} G\left(t-t^{\prime}\right) \mathcal{F}_{A}\left(t^{\prime}\right) \mathrm{d} t^{\prime},
\end{aligned}
$$

implying that the temperature starts changing in response to this forcing from a non-equilibrium initial state. However, the statistics of volcanic forcing is more challenging than assumed above, and one has to consider the possibility of long periods with zero forcing, longer than the largest temperature relaxation time reflected in the response function $G(t)$. If such a quiet period starts at time $t_{q}$, then the temperature for $t>t_{q}$ is

$$
\Delta T(t)=\overline{\mathcal{F}}_{V} \int_{t-t_{q}}^{\infty} G(s) \mathrm{d} s+\int_{0}^{t} G\left(t-t^{\prime}\right) \mathcal{F}_{A}\left(t^{\prime}\right) \mathrm{d} t^{\prime},
$$

and since the integral over the tail of $G(s)$ is assumed to be finite (there exists a maximum relaxation time constant $\tau_{\max }$ ), the first term on the right of Eq. (E15) will vanish if $t>t_{q}+\tau_{\max }$. In other words, if the time of observation has been preceded by a very long period of weak volcanic forcing the additionally forced temperature change may be unaffected by the non-equilibrium imposed by volcanic forcing. If we consider, as another example, that "normal" volcanic forcing is resumed at $t=0$ after a pause of the length of $\left|t_{q}\right|>\tau_{\max }$, then $\Delta T$ according to Eq. (E15) grows from zero towards the expression in Eq. (E14) as $t$ grows beyond $t_{\max }$. Hence, during the transient period $t \in\left(0, \tau_{\max }\right)$ there may be a volcanic cooling that counteracts anthropogenic warming, provided there was a long pause in volcanic forcing preceding the era of anthropogenic forcing.
The discussion made here serves to illustrate that the nonequilibrium of the radiative flux balance at $t=0$ may influence the subsequent temperature evolution and that volcanic forcing may be the source of such an imbalance. Knowledge about the history of volcanic forcing in the time interval $\left(-\tau_{\max }, t\right)$ can be helpful in assessing the influence of volcanic forcing on the long-term temperature evolution in the Anthropocene. In the present paper the implicit assumption has been made that Eq. (E14) is valid, i.e. that there is no long pause in volcanic forcing in the period extending from $1880-\tau_{\max }$ to $2200 \mathrm{CE}$. Hence, this forcing only represents a constant downshift of the temperature. This assumption may deserve closer scrutiny.

\section{Appendix F: Non-stationarity of the $\mathrm{CO}_{2}$ response}

In Sect. 2.2 we found (by comparing Fig. $4 \mathrm{~b}$ and c) that the LRM $\mathrm{CO}_{2}$ response with $\beta_{\mathrm{C}}=1.6$ gives approximately the same evolution of $\mathrm{CO}_{2}$ concentration up to $2200 \mathrm{CE}$ as a response where $50 \%$ of the emitted $\mathrm{CO}_{2}$ is absorbed by the surface almost immediately and the remainder decays exponentially with a time constant $\tau_{\mathrm{C}}=300$ years. This is analogous to the situation with the temperature response, where an LRM response gives very similar results to a two-exponential response with appropriate fitting of model parameters (see Appendix D). The most important difference is that the $\beta_{\mathrm{C}}$ parameter is larger than unity. A step-function emission rate $R(t)=H(t)$ will give rise to a $\mathrm{CO}_{2}$ concentration that grows like $\left(2 \alpha_{T} / \beta_{\mathrm{C}}\right) t^{\beta_{\mathrm{C}} / 2}$. This non-stationarity (divergence) of the response as $t \rightarrow \infty$ is reasonable, since the surface will not be able to absorb a sufficient fraction of the constantly emitted $\mathrm{CO}_{2}$ to establish a new equilibrium. The exponential response kernel Eq. (6), on the other hand, yields the response $r\left[1-\exp \left(-t / \tau_{\mathrm{C}}\right)\right]$ to the step forcing. This implies the establishment of a new equilibrium $\mathrm{CO}_{2}$ concentration after $t \gg \tau_{\mathrm{C}}$. This has little consequence as long as we consider projection only up to $2200 \mathrm{CE}$ (and $\tau_{\mathrm{C}} \approx 300$ years). On a millennium timescale we have the positive ice-age feedback, by which warming may lead to net release of $\mathrm{CO}_{2}$ to the atmosphere, and hence lead to a continuing growth of $\mathrm{CO}_{2}$ concentration. It is assumed to be important in the triggering of glacial-interglacial transitions, although it is not very well understood. On timescales of hundreds of kiloyears, we have the negative carbon-weathering-cycle feedback that will eventually lead to a carbon-cycle equilibrium. The most interesting feature of this feedback in the present context is that it suggests that the anthropogenic global warming event may last for such a long time in the absence of effective carbon sequestration measures (Archer, 2010).

A more problematic non-stationarity of the carbon-cycle response arises from stochastic forcing. In this case the power-law response function will give rise to a fractional Brownian motion (fBm) with power-spectral index $\beta_{\mathrm{C}} \approx$ 1.6. This is a non-stationary stochastic process in the sense 
that the variance increases with time as $t^{\beta_{\mathrm{C}}-1}$, which is not physically reasonable for sufficiently large $t$. Here, we may be saved by an exponential cut-off of the power-law tail, but this requires some sort of negative carbon-cycle feedback. It is difficult to assess the magnitude of the natural stochastic component of the $\mathrm{CO}_{2}$ emission rate. If it is small enough, the weathering-cycle feedback may be sufficient. 


\section{The Supplement related to this article is available online at doi:10.5194/esd-7-51-2016-supplement.}

Acknowledgements. This work was funded by project no. 229754 under the Norwegian Research Council KLIMAFORSK programme.

Edited by: R. Krishnan

\section{References}

Archer, D.: The Global Carbon Cycle, Princeton Primers in Climate, Princeton University Press, Princeton, Chapter 12, 287295, 2010

Allen, M. R., Frame, D. J., Huntingford, C., Jones, C. D., Lowe, J. A., Meinshausen, M., and Meinshausen, N.: Warming caused by cumulative carbon emissions towards the trillionth tonne, Nature, 458, 1163-1166, doi:10.1038/nature08019, 2009.

Anderegg, W. R. L., Prall, J. W, Harold, J., and Schneider, S. H.: Expert credibility in climate change, P. Natl. Acad. Sci., 107, 12107-12109, doi:10.1073/pnas.1003187107, 2010.

Bender, M. L.: Paleoclimate, Princeton Primers in Climate, Princeton University Press, Princeton, Chapter 10, 235-263, 2013.

Beran, J.: Statistics for Long-memory Processes, Monographs on statistics and applied probability, Chapman\& Hall/CRC, Boca Raton, 1994.

Brohan, P., Kennedy, J. J., Harris, I., Tett, S. F. B., and Jones, P. D.: Uncertainty estimates in regional and global observed temperature changes: A new data set from 1850, J. Geophys. Res, 111, D12106, doi:10.1029/2005JD006548, 2006.

Budyko, M. I.: The effect of solar radiation variations on the climate of the Earth, Tellus, 21, 611-619, 1969.

Cook, J., Nuccitelli, D., Green, S. A., Richardson, M., Winkler, B., Painting, R., Way, R., Jacobs, P., and Skuce, A. : Quantifying the consensus on anthropogenic global warming in the scientific literature, Environ. Res. Lett. 8, 024024, doi:10.1088/17489326/8/2/024024, 2013.

den Elzen, M., Meinshausen, M., and van Vuuren, D.: Multigas emission envelopes to meet greenhouse gas concentration targets: costs versus certainty of limiting temperature increase, Global Environ. Change, 17, 260-280, doi:10.1016/j.gloenvcha.2006.10.003, 2007.

Geoffroy, O., Saint-Martin, D., Olivié, D. J. L., Voldoire, A., Bellon, G., and Tytcá, S.: Transient Climate Response in a Two-Layer Energy-Balance Model. Part I: Analytical Solution and Parameter Calibration Using CMIP5 AOGCM Experiments, J. Climate, 6, 1841-1857, doi:10.1175/JCLI-D-12-00195.1, 2013.

Fraedrich, K. and Blender, R.: Scaling of Atmosphere and Ocean Temperature Correlations in Observations and Climate Models, Phys. Rev. Lett., 90, 108501-10854, doi:10.1103/PhysRevLett.90.108501, 2003.

Granger, C. W. J.: Long Memory Relationships and the aggregation of dynamical models, J. Econometrics, 14, 227-238, 1980.

Hansen, J., Sato, M., Kharecha, P., and von Schuckmann, K.: Earth's energy imbalance and implications, Atmos. Chem. Phys., 11, 13421-13449, doi:10.5194/acp-11-13421-2011, 2011.
Lenton, T. M., Held, H., Kriegler, E., Hall J. W., Lucht, W., Rahmstorf, S., and Schellnhuber, H. J.: Tipping elements in the Earth's climate system, P. Natl. Acad. Sci., 105, 1786-1793, doi:10.1073/pnas.0705414105, 2008.

Lovejoy, C., Schehrtzer, D., and Varon, D.: Do GCMs predict climate... or macroweather?, Earth Syst. Dynam., 4, 439-454, doi:10.5194/esd-4-439-2013, 2013.

Stocker, T. F., Qin, D., Plattner, G.-K., Tignor, M., Allen, S. K., Boschung, J., Nauels, A., Xia, Y., Bex, V., and Midgley, P. M. (Eds.).: IPCC, 2013: Climate Change 2013: The Physical Science Basis. Contribution of Working Group I to the Fifth Assessment Report of the Intergovernmental Panel on Climate Change, Cambridge University Press, Cambridge, UK and New York, NY, USA, 1535 pp., 2013.

Joos, F., Roth, R., Fuglestvedt, J. S., Peters, G. P., Enting, I. G., von Bloh, W., Brovkin, V., Burke, E. J., Eby, M., Edwards, N. R., Friedrich, T., Frölicher, T. L., Halloran, P. R., Holden, P. B., Jones, C., Kleinen, T., Mackenzie, F. T., Matsumoto, K., Meinshausen, M., Plattner, G.-K., Reisinger, A., Segschneider, J., Shaffer, G., Steinacher, M., Strassmann, K., Tanaka, K., Timmermann, A., and Weaver, A. J.: Carbon dioxide and climate impulse response functions for the computation of greenhouse gas metrics: a multi-model analysis, Atmos. Chem. Phys., 13, 2793 2825, doi:10.5194/acp-13-2793-2013, 2013.

Le Quéré, C., Moriarty, R., Andrew, R. M., Peters, G. P., Ciais, P., Friedlingstein, P., Jones, S. D., Sitch, S., Tans, P., Arneth, A., Boden, T. A., Bopp, L., Bozec, Y., Canadell, J. G., Chevallier, F., Cosca, C. E., Harris, I., Hoppema, M., Houghton, R. A., House, J. I., Jain, A., Johannessen, T., Kato, E., Keeling, R. F., Kitidis, V., Klein Goldewijk, K., Koven, C., Landa, C. S., Landschützer, P., Lenton, A., Lima, I. D., Marland, G., Mathis, J. T., Metzl, N., Nojiri, Y., Olsen, A., Ono, T., Peters, W., Pfeil, B., Poulter, B., Raupach, M. R., Regnier, P., Rödenbeck, C., Saito, S., Salisbury, J. E., Schuster, U., Schwinger, J., Séférian, R., Segschneider, J., Steinhoff, T., Stocker, B. D., Sutton, A. J., Takahashi, T., Tilbrook, B., van der Werf, G. R., Viovy, N., Wang, Y.-P., Wanninkhof, R., Wiltshire, A., and Zeng, N.: Global carbon budget 2014, Earth Syst. Sci. Data Discuss., 7, 521-610, doi:10.5194/essdd-7-521-2014, 2014.

Matthews, H. D., Gillett, M. P., Stott, P. A., and Zickfeld, K.: The proportionality of global warming to cumulative carbon emissions, Nature, 459, 1129-132, doi:10.1038/nature08047, 2009.

Meehl, G. A., Washington,W. M., Amman,C. M., Arblaster, J. M., Wigley, T. M., and Tebaldi, C.: Combinations of Natural and Anthropogenic Forcings in Twentieth-Century Climate, J. Climate, 17, 3721-3727, 2004.

Meinshausen, M., Smith, S. J., Calvin, K., Daniel, J. S., Kainuma, M. L. T., Lamarque, J.-F., Matsumoto, K., Montzka, S. A., Raper, S. C. B., Riahi, K., Thomson, A., Velders, G. J. M., van Vuuren, D. P. P.: The RCP greenhouse gas concentrations and their extensions from 1765 to 2300, Clim. Change, 11, 2013-241, doi:10.1007/s10584-011-0156-z, 2011.

Myhre, G., Highwood, J., Shine, K. P., and Stordahl, F.: New estimates of radiative forcing due to well-mixed greenhouse gases, Geophys. Res. Lett., 25, 2715-2718, 1998.

Østvand, L., Nilsen, T., Rypdal, K., Divine, D., and Rypdal, M.: Long-range memory in internal and forced dynamics of millennium-long climate model simulations, Earth. Syst. Dynam., 5, 295-308, doi:10.5194/esd-5-295-2014, 2014. 
Rypdal, K.: Global temperature response to radiative forcing: Solar cycle versus volcanic eruptions, J. Geophys. Res., 117, D06115, doi:10.1029/2011JD017283, 2012.

Rypdal, M. and Rypdal, K.: Long-memory effects in linearresponse models of Earth's temperature and implications for future global warming, J. Climate, 27, 5240-5258, doi:10.1175/JCLI-D-13-00296.1, 2014.

Rypdal, K., Rypdal, M., and H.-B. Fredriksen: Spatiotemporal Long-Range Persistence in Earth's Temperature Field: Analysis of Stochastic-Diffusive Energy Balance Models, J. Climate, 28, 8379-8395, doi:10.1175/JCLI-D-15-0183.1, 2015.

Schwartz, S. E.: Heat capacity, time constant, and sensitivity of the Earth's climate system, J. Geophys. Res., 112, D24S05, doi:10.1029/2007JD008746, 2007.
Sellers, W. D.: A global climate model based on the energy balance of the Earth-atmosphere system, J. Appl. Meteorol., 8, 392-400, 1969.

Solomon, L.: The Deniers. The world-renowned scientists who stood up against global warming hysteria, political persecution and fraud, Richard Viglante Books, 1-239. 2008.

Stocker, T.: The Closing Door of Climate Targets, Science, 339, 280-282, doi:10.1126/science.1232468, 2013.

Stern, N.: The Economics of Climate Change. The Stern Review, Cambridge, 1-673, 2007. 